\title{
Updating 3D Planning Data based on Detected Differences between Real and Planning Data of Building Interiors
}

\author{
Andreas Dietze \\ Fulda University of \\ Applied Sciences \\ Leipziger Straße 123 \\ 36037 Fulda, Germany \\ andreas.dietze@cs.hs- \\ fulda.de
}

\author{
Paul Grimm \\ Darmstadt University of \\ Applied Sciences \\ Haardtring 100 \\ 64295 Darmstadt, \\ Germany \\ paul.grimm@h-da.de
}

\author{
Yvonne Jung \\ Fulda University of \\ Applied Sciences \\ Leipziger Straße 123 \\ 36037 Fulda, Germany \\ yvonne.jung@cs.hs- \\ fulda.de
}

\begin{abstract}
This paper presents a system to determine differences between 3D reconstructed interiors and their corresponding 3D planning data with the aim of correcting identified differences and updating the 3D planning data based on these deviations. Therefore, a point-based comparison algorithm was developed with which deviations can be recognized regardless of the topology of the data used. Usually, resolution and topology of a 3D reconstruction do not match the CAD data. Here, our solution overcomes this problem by segmenting and extracting objects relevant for comparison (e.g., doors, windows) from the reconstruction and planning data separately with a subsequent analysis of the proximity of these objects to connected walls within the corresponding data set. Starting from the connection points of a segmented object to its walls, adjacent spatial data is located for a correction of detected differences to update the 3D planning data. The quality of the result of the developed process is shown in different examples localizing doors and windows to find deviations. In addition, detected differences between the planning and the measurement data are visualized and compared with the ground truth state of the building interior.
\end{abstract}

\section{Keywords}

3D Geometry Comparison, Geometry Deviation Feedback, Object Recognition, Computer Vision, Point Clouds, Mobile Mixed Reality

\section{INTRODUCTION}

In many industrial and commercial areas, Mixed Reality techniques are nowadays used in the planning or production process as well as for construction monitoring and quality control, e.g. to discover deviations that arise during a construction process at an early stage. If such deviations cannot be fixed, this could be a problem for later maintenance. Thus, our approach allows an update of the planning data respectively. Especially an early detection of differences to the original blueprints, like shifted windows, doors, or walls, can help to reduce or avoid high follow-up costs and support adherence to the schedules. This also involves a modernization or redesign of existing buildings or interiors, for example when doors are newly set or sealed. While other systems for digital construction monitoring are often limited to a visualization of the monitoring and docu-

Permission to make digital or hard copies of all or part of this work for personal or classroom use is granted without fee provided that copies are not made or distributed for profit or commercial advantage and that copies bear this notice and the full citation on the first page. To copy otherwise, or republish, to post on servers or to redistribute to lists, requires prior specific permission and/or a fee. mentation progress $\left[\mathrm{ZHK}^{+} 14\right.$, GFPMS09] or a visualization and comparison of detected differences between measurement and planning data [Bos10, DFFW18], the focus of the system presented in this paper, in addition to detect, visualize, and compare differences $\left[\mathrm{KER}^{+} 17\right.$, DGJ20], is to correct differences with a subsequent feedback into the 3D planning data.

Acquiring the depth data for a $3 \mathrm{D}$ reconstruction can be performed in various ways, such as by photogrammetry (Structure from Motion), Time of Flight, or Structured Light $\left[\mathrm{LJS}^{+} 20\right]$. Determined spatial data is represented in the form of $3 \mathrm{D}$ point clouds or a $3 \mathrm{D}$ model is constructed from the point cloud in further postprocessing $\left[\mathrm{GSC}^{+} 07, \mathrm{NIH}^{+} 11\right]$. Various analytical, region-based and geometric methods (model fitting) are used for cluster analysis of point clouds, in particular for segmenting and extracting certain elements of a scene [GMR17, $\left.\mathrm{SWL}^{+} 16\right]$. In addition, also more and more approaches based on machine learning are applied [ZLY19]. For a comparison of 3D data, Doboš et al. [DFFW18] described a method that recognizes differences between 3D models in the screen space based on different data such as color, depth, normals and texture coordinates and visualizes them for the user. Furthermore, Tuttas et al. [TBBS14, TSBB15, TBBS17] de- 
scribed an approach that compares point clouds from real scenes with planning data in order to enable automated building documentation. The point clouds are recorded by photogrammetry and Structure from Motion. The main contribution of this paper is an algorithm that detects deviations from planning data compared to scanning data from the real world either to correct the deviations at the construction site or to use this information to update the planning data. In the latter case the differences found are used to adjust the original planning data respectively (e.g., for maintenance). Therefore, differences between planning data and captured real data are compared using a point-cloud-based data representation and the resulting differences are used to change the planning data on a geometry level.

\section{PREPROCESSING}

The first task of the whole system pipeline is to clean up the captured data. In our case the capturing is carried out using a Microsoft HoloLens, but our system is not limited to it, so that other 3D scanners, for example Occipital's Structure Sensor ${ }^{1}$ for an Apple iPad, could be used. During this preprocessing, the captured point cloud is cleared of outliers and points that have arisen due to a misinterpretation of the depth data, which for example can occur due to highly reflective or transparent surfaces. After this preprocessing the identification, localization, segmentation, and extraction of objects relevant for comparison is carried out. The removal of outliers that are not part of the interior of the 3D reconstruction is achieved by using a mass histogram and the determined amplitudes. First, the orthographic projection of the point cloud is divided into individual segments of fixed size along a unit axis. Localized points within the boundary of a segment are added to a subset determining the distribution of points along that axis. Using both highest amplitudes in the histogram, the point cloud can be freed from outliers, as illustrated in figure 1 and shown in listing 1.

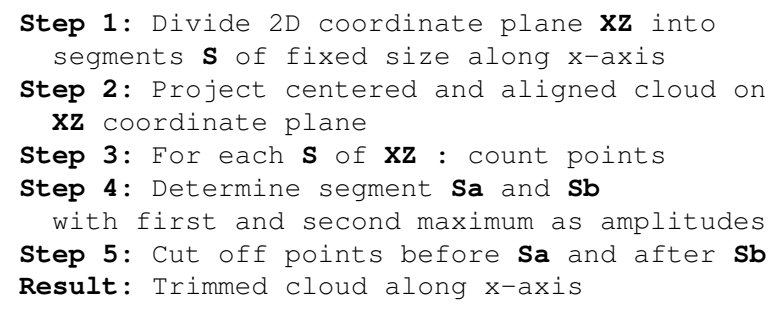

Listing 1: Outlier removal using the mass histogramm and both determined amplitudes along the $\mathrm{x}$-axis. The same process is also done for $\mathrm{y}$ - and $\mathrm{z}$-axis.

During the reconstruction process, objects that are relevant for comparison, such as windows or doors, may

1 Occipital Structure Sensor https://structure.io/ be occluded or have semi-transparent or partially intermediate regions, which makes it more difficult or even impossible to segment these objects later on for the purpose of detecting differences. If available, spatial information about such objects, like fitted units, can be obtained by the BIM (Building Information Modeling) data of a building or have to be measured manually. Another solution could be using a CNN, which identifies and locates even partwise occluded windows and doors to provide metadata of the located objects. Within our semi-automatic solution, the developed scanning software allows marking occluded regions during the reconstruction process, which can help to remove outliers within these regions and to free up occluded objects. The left side of figure 2 illustrates the reconstruction from the user's point of view including the regions marked for a later clean up. Based on the corner points of a marked region, a cuboid with twice the depth of the width of a corresponding region (negative and positive depth along the plane's normal) is used to remove points within its boundarys, which is illustrated on the right side of figure 2 .

Regions marked are saved as metadata in a separate file related to the $3 \mathrm{D}$ model of the $3 \mathrm{D}$ reconstruction. The metadata contains a clear identification of the sliced object, as well as the object type, which provides information on whether the cut out object is a door or a window. Furthermore, the geometry type keeps information about what shape the user used to cut out the object, which he had previously selected from a range of predefined shapes (circle, rectangle, polygon). The anchor count contains the number of points used for the geometry type and the anchor points are stored as linear vector that contains the actual spatial data. In addition, the surface normal of the cut out geometry and the estimated unit normal are stored. Based on the anchor points and the unit normal, the dimensions of the cuboid are set up. Figure 3 contains a cut out from the metadata contained in the file, which represents the metadata related to the window within the right side of figure 2 . Figure 4 shows the 3D reconstruction in form of the raw point cloud (left) and the preprocessed point cloud (right), in which all points within the regions previously marked were removed, as well as a removal of outliers based on the mass histogram has been carried out (see figure 1). Also mentionable is the variable depth of cuboids using the determined width based on the planes created from the metadata for freeing occluded regions, which enables filtering doors or windows that are opened to the interior, as it can be seen in the right lower parts of figure 4 .

\section{DETECTING DIFFERENCES}

To identify and locate differences between planning and actual data, segmentation and extraction of objects relevant for comparison is done individually for both 

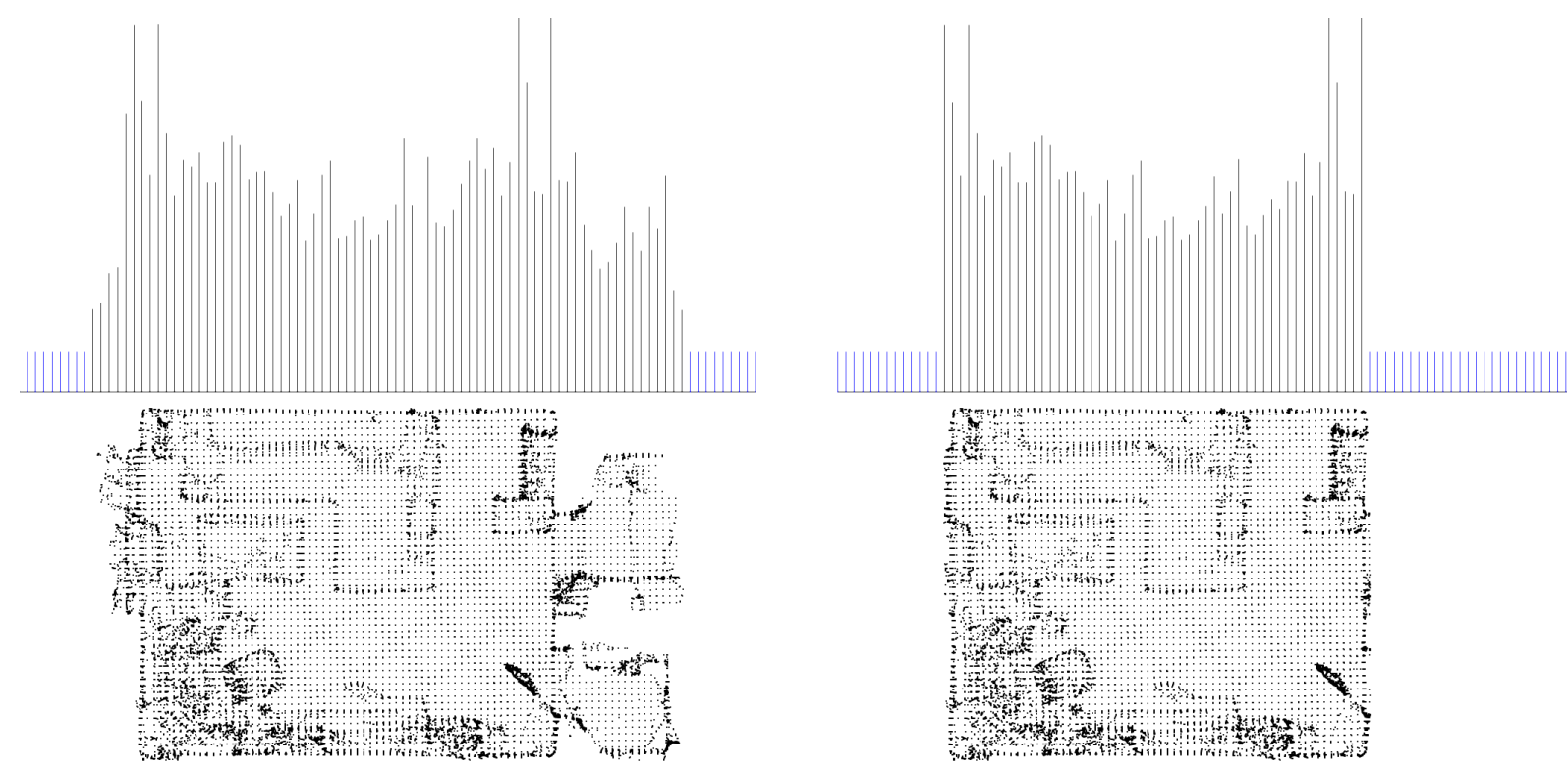

Figure 1: Left: mass histogram and corresponding point cloud. Right: point cloud cleaned of outliers based on determined amplitudes in the mass histogram along $\mathrm{x}$-axis.
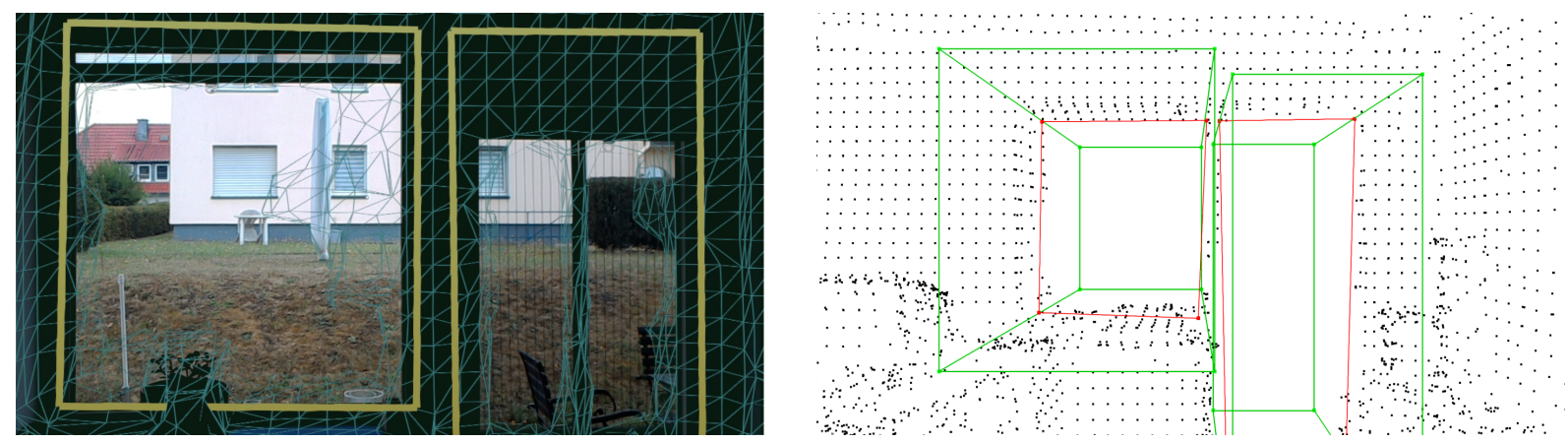

Figure 2: Creation of metadata during the 3D reconstruction process. The user marks regions that are interpreted as part of the interior due to misinterpreted depth data or occluded regions (left). The metadata created is used to remove outliers and points that have arisen due to misinterpreted depth data or that occlude objects relevant for a comparison (right).

\# Sliced Object Data (.sod)

\# Used as metadata for window or door objects in SFRA. \#\#\#\#\#\#\# Content \#\#\#\#\#\#\#

SLICED_OBJECTS

Socount : 5

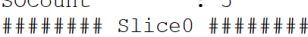

Id : Sliceo

objectrype : Window

GeometryType : Rectangle

Anchorcount : 4

AnchorPoints : $0.3602401,0.9329683,-3.7518,-0.506594, \ldots$

FaceNormal : $0.04176028,-0.03948342,-0.9983472$

UnitNormal : $0,0,1$

Figure 3: Example of the created metadata, which is optionally created during the reconstruction process and stored separately for the related $3 \mathrm{D}$ model of the reconstruction.

datasets. For the planning data segmentation, the 2D floor plan is converted into a planar point cloud and the wall segments are localized and extracted by use of a Euclidian Cluster Extraction (ECE) ${ }^{2}$. Each individual wall segment is connected to its both nearest neighbours and the located regions between two wall seg-

2 Euclidean Cluster Extraction https://pcl.readthedocs.io/en/ latest/cluster_extraction.html ments identify windows or doors as illustrated in figure 5. The left part of the figure shows the extracted ground segment. In the right part of the figure, walls, windows, and doors were segmented and extracted from the planar point cloud of the converted floor plan. Within the reconstructed point cloud, all wall faces are filtered and extracted using the amplitudes found in the mass histogram, as illustrated on the left in figure 6. Subsequently, openings are localized in the planar point cloud of the extracted wall side using $\alpha$-shapes [TC98], as visualized on the right in figure 6 . Figure 7 visualizes an overlay of all segmented and extracted objects relevant in the context of a difference detection in addition to the planar point cloud of the floor plan and the preprocessed point cloud of the reconstruction. Table 1 provides a comparison of the found differences between the planning data and its reconstruction concerning the width. As can be seen, differences between all extracted objects have been detected but the difference calculated for door 3 in table 1 is particularly noticeable compared to the other results. The reason for the high deviation 

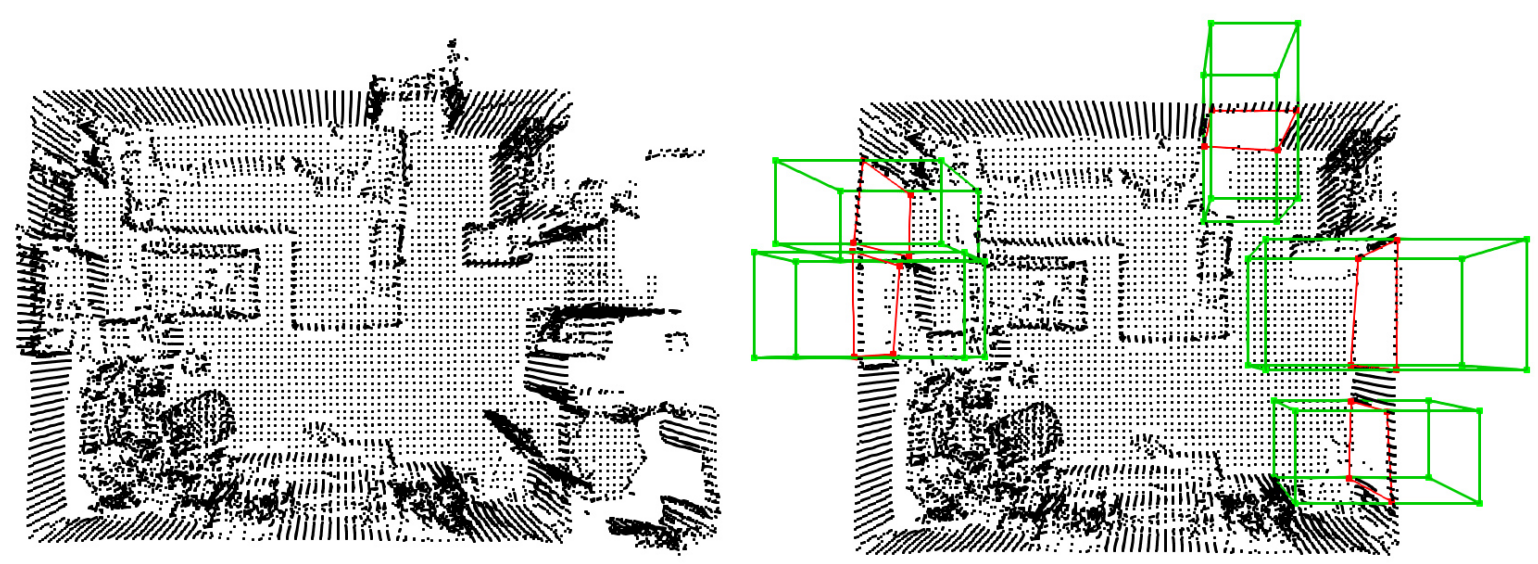

Figure 4: On the left side, the raw $3 \mathrm{~d}$ reconstruction is visualized as point cloud. On the right, the preprocessed point cloud is visualized, using the mass histogramm to remove outliers and the metadata to clean up occluded regions.

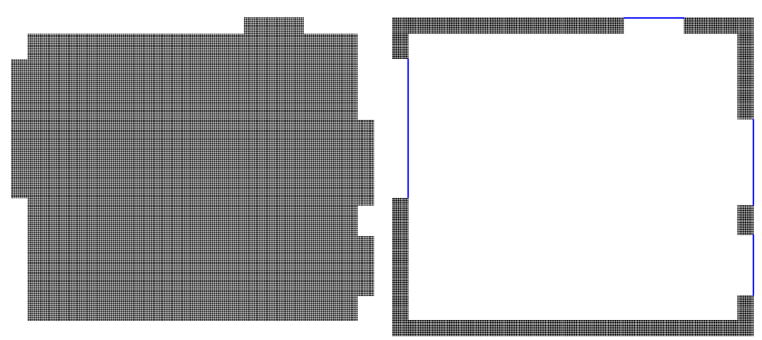

Figure 5: Planar point cloud of the floor plan split up into the extracted ground segment (left) and the individual wall segment point clouds (right) using an ECE. Each interconnection between two wall segments locates the start and end point of a window or door object within the 2D planning data.
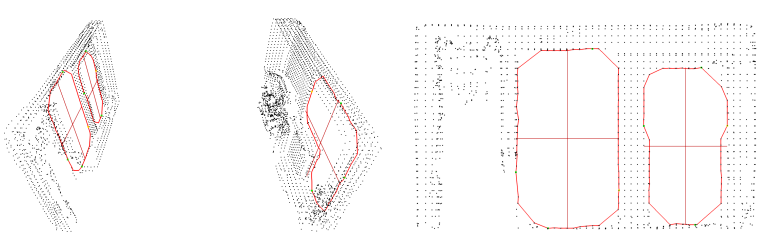

Figure 6: Localization of relevant objects for a comparison of differences using $\alpha$-shapes in the planar point cloud of the extracted wall sides by the amplitudes of the mass histogram.

\begin{tabular}{|c|c|c|c|c|}
\hline & Door 1 & Window 1 & Door 2 & Door 3 \\
\hline Target & $0.89 \mathrm{~m}$ & $2.04 \mathrm{~m}$ & $1.26 \mathrm{~m}$ & $0.89 \mathrm{~m}$ \\
\hline Actual & $0.93 \mathrm{~m}$ & $2.01 \mathrm{~m}$ & $1.29 \mathrm{~m}$ & $1.04 \mathrm{~m}$ \\
\hline Difference & $0.04 \mathrm{~m}$ & $0.03 \mathrm{~m}$ & $0.03 \mathrm{~m}$ & $0.15 \mathrm{~m}$ \\
\hline Compare & $\pm 4.30 \%$ & $\pm 1.49 \%$ & $\pm 2.32 \%$ & $\pm 14.42 \%$ \\
\hline
\end{tabular}

Table 1: Comparison of differences detected between planning and actual data in context of width.

is misinterpreted depth data that occurred during the scanning process, as can be seen from the mushroomshaped upper part in the right door within the right side of figure 6. In addition, table 2 and table 3 are containing a comparison between the planning data, the real state of the building interior (ground truth) and the reconstruction.

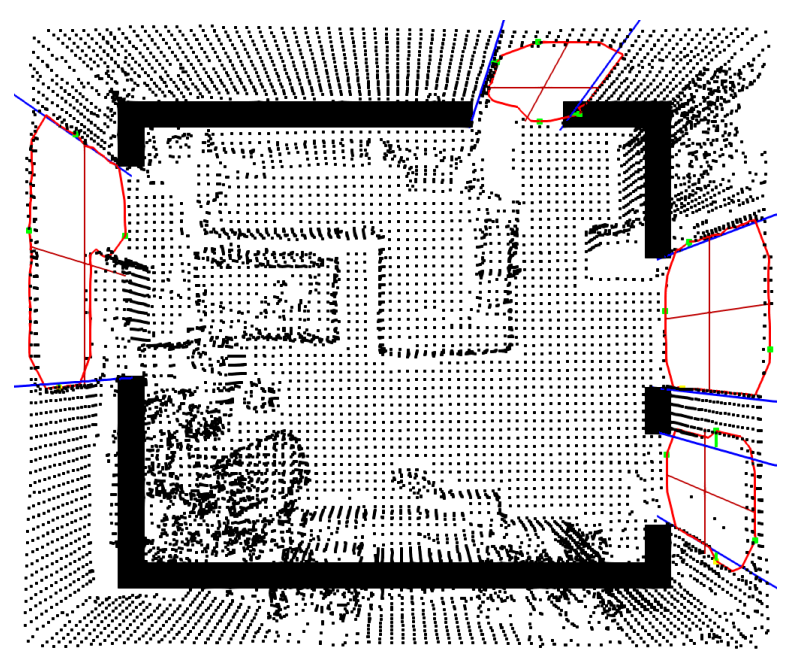

Figure 7: Overlay of the extracted regions from the planar point cloud of the floor plan by ECE (blue lines), the extracted wall segments from the floor plan point cloud (black rectangles), the preprocessed point cloud of the reconstruction as well as the extracted polygons from the segmented wall sides using $\alpha$-shapes (red).

\begin{tabular}{|c|c|c|c|c|}
\hline & Door 1 & Window 1 & Door 2 & Door 3 \\
\hline Target & $0.89 \mathrm{~m}$ & $2.04 \mathrm{~m}$ & $1.26 \mathrm{~m}$ & $0.89 \mathrm{~m}$ \\
\hline Real & $0.91 \mathrm{~m}$ & $2.05 \mathrm{~m}$ & $1.25 \mathrm{~m}$ & $0.90 \mathrm{~m}$ \\
\hline Difference & $0.02 \mathrm{~m}$ & $0.01 \mathrm{~m}$ & $0.01 \mathrm{~m}$ & $0.01 \mathrm{~m}$ \\
\hline Compare & $\pm 2.19 \%$ & $\pm 0.48 \%$ & $\pm 0.80 \%$ & $\pm 1.11 \%$ \\
\hline
\end{tabular}

Table 2: Comparison of differences between planning data and real state in context of width.

\begin{tabular}{|c|c|c|c|c|}
\hline & Door 1 & Window 1 & Door 2 & Door 3 \\
\hline Real & $0.91 \mathrm{~m}$ & $2.05 \mathrm{~m}$ & $1.25 \mathrm{~m}$ & $0.90 \mathrm{~m}$ \\
\hline Actual & $0.93 \mathrm{~m}$ & $2.01 \mathrm{~m}$ & $1.29 \mathrm{~m}$ & $1.04 \mathrm{~m}$ \\
\hline Difference & $0.02 \mathrm{~m}$ & $0.04 \mathrm{~m}$ & $0.04 \mathrm{~m}$ & $0.14 \mathrm{~m}$ \\
\hline Compare & $\pm 2.15 \%$ & $\pm 1.99 \%$ & $\pm 3.10 \%$ & $\pm 13.46 \%$ \\
\hline
\end{tabular}

Table 3: Comparison of differences between real state and actual data in context of width. 


\section{UPDATING 3D PLANNING DATA}

To be able to feed back detected differences into the 3D planning data, the corresponding points of a wall segment are localized for which a difference of the counterpart from the $3 \mathrm{D}$ reconstruction was found. Since differences are initially detected based on the determined deviations between doors and windows, these differences between two corresponding objects are used to manipulate the wall segments in the $3 \mathrm{D}$ planning data. If, for example, a discrepancy between a door from the planning data and the associated door from the spatial data of the reconstruction has been determined, points of the wall segments from the $3 \mathrm{D}$ planning data that are connected to this door are manipulated. For this purpose, all points of a wall segment that are related to a correction of the detected difference are first to be localized. This is made possible by using the adjacent properties of the individual wall segments and the connection points of two wall segments that are connected by the door or window in between. Starting from a connection point, points that are relevant for the manipulation can be localized by performing a collision detection for corner points that are located near to the location of the connection point with an intersection shape (e.g., circle or rectangle) on the planar point cloud of the planning data. Figure 8 illustrates the process.

As intersection shape a rectangular shape was used to determine the points that belong to a wall segment and are being part of the edge of this wall segment. The width and height for a rectangle is in correlation to the thickness of a wall segment and the detected difference to this location. For a better visualization, a fixed width and height was used to illustrate the localization of points relevant for a correction.

Relevant points for a correction of the determined differences were localized using the procedure described in the previous section. In the next step, the differences determined in section 3 can be fed back automatically and persistently into the 3D planning data. For this purpose, all points determined on one side of a wall segment are transformed according to the difference found. Figure 9 shows the correction of the spatial data determined based on the regions in figure 8 by an overlay of the $3 \mathrm{D}$ planning data before and after the correction of differences. Listing 2 shows the procedure in summary.

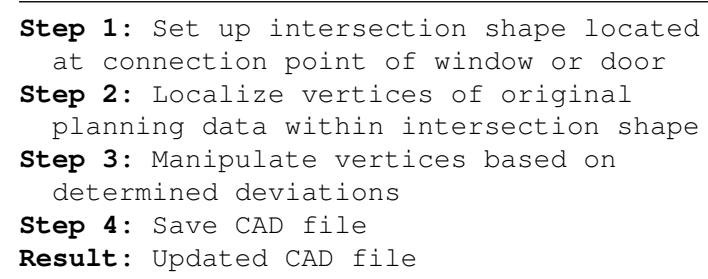

Listing 2: Correction of original CAD data based on detected differences.

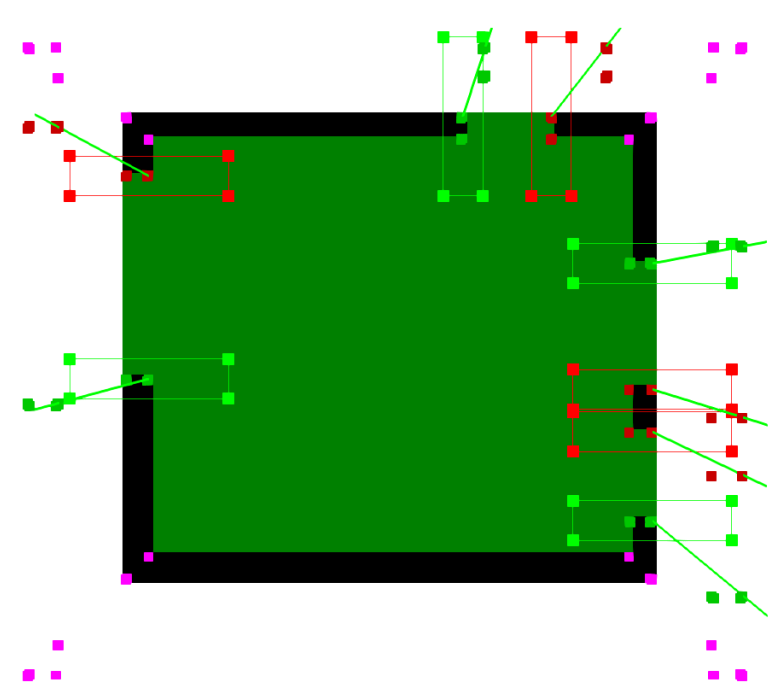

Figure 8: 2D planning data and the determined points of the 3D planning data for a correction of deviations. Points within an intersection shape belong to the wall segment that is connected to its neighboring wall segments by the window or door object in between and its starting point (green points within the green intersection shapes) and end point (red points within red intersection shapes). Purple points are not relevant for a correction of deviations.

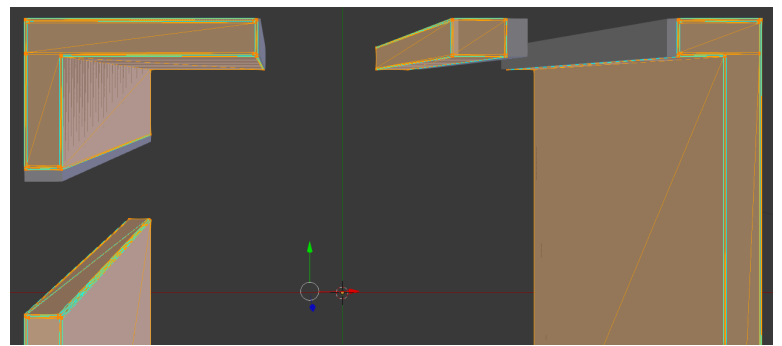

Figure 9: Overlay of the 3D planning data as 3D models before (grey) and after (yellow) the correction of differences. The view represents the $3 \mathrm{D}$ model of the planning data equivalent to the $2 \mathrm{D}$ and $3 \mathrm{D}$ planning data illustrated in figure 8 .

\section{CONCLUSION AND OUTLOOK}

In this paper, we have presented a system, which allows an automated detection and visualization of differences between a $3 \mathrm{D}$ reconstruction of interiors and their corresponding planning data. Our proposed system is also capable of feeding back detected differences persistently into the 3D planning data. Furthermore, the preprocessing including the removal of outliers has been improved by using the metadata created during the reconstruction process with the Microsoft HoloLens to free occluded regions from points, which are not part of the object's structure.

For future work, we would firstly like to incorporate more complex types of geometries. Due to the system's actual limitation to detect and correct differences within manhattan world environments, next steps could focus on the possibility to handle more complex shaped rooms, which, for example, could be made possible by 
using the floor plan point cloud for an outlier removal. In addition, further optimization and comparisons concerning the accuracy are possible. Secondly, we'd like to focus on visualization aspects, where a meta file format similar to the metadata created during the reconstruction process can be used that provides all neccessary data for a visualization of detected differences on the HoloLens and other Augmented or Mixed Reality devices. Moreover, such an AR-based difference visualization, maybe even annotated with further information about the corresponding problem, would greatly enhance on-site discussions during the building phase as well as later maintainance tasks.

\section{REFERENCES}

[Bos10] F. Bosché. Automated recognition of 3d cad model objects in laser scans and calculation of as-built dimensions for dimensional compliance control in construction. Advanced Engineering Informatics, 24:107-118, 012010.

[DFFW18] J. Doboš, C. Fan, S. Friston, and C. Wong. Screen space $3 \mathrm{~d}$ diff: A fast and reliable method for real-time $3 \mathrm{~d}$ differencing on the web. In Proc. of the 23rd Intl. Conf. on 3D Web Technology, Web3D '18, New York, USA, 2018. ACM.

[DGJ20] A. Dietze, P. Grimm, and Y. Jung. Visualization of differences between spatial measurements and $3 \mathrm{~d}$ planning data. In Proc. of the 25th Intl. Conf. on 3D Web Technology, pages 1-5. ACM, 2020.

[GFPMS09] M. Golparvar-Fard, F. Pena-Mora, and S. Savarese. Application of d4ar - a 4dimensional augmented reality model for automating construction progress monitoring data collection, processing and communication. Journal of Information Technology in Construction, 14:129-153, 062009.

[GMR17] E. Grilli, F. Menna, and F. Remondino. A review of point clouds segmentation and classification algorithms. ISPRS - International Archives of the Photogrammetry, Remote Sensing and Spatial Information Sciences, XLII-2/W3:339-344, 2017.

$\left[\mathrm{GSC}^{+} 07\right]$ M. Goesele, N. Snavely, B. Curless, H. Hoppe, and M. Seitz, S. Multi-view stereo for community photo collections. In 2007 IEEE 11th Intl. Conference on Computer Vision, pages 1-8, 2007.

$\left[\mathrm{KER}^{+}{ }^{17]}\right.$ M. Klomann, M. Englert, A. Rehberger, A. Dietze, T. Geier, S. Rieger, P. Grimm, and Y. Jung. NetFlinCS: A hybrid cloudbased framework to allow context-based detection and surveillance. In Proceedings VSMM '17, pages 1-8. IEEE, 2017.

$\left[\mathrm{LJS}^{+} 20\right]$ Y. Liu, J. Jiang, J. Sun, L. Bai, and Q. Wang. A survey of depth estimation based on computer vision. In 2020 IEEE 5th Intl. Conference on Data Science in Cyberspace, pages 135-141, 2020.

$\left[\mathrm{NIH}^{+} 11\right]$ R. A. Newcombe, S. Izadi, O. Hilliges, D. Molyneaux, D. Kim, A. J. Davison, P. Kohi, J. Shotton, S. Hodges, and A. Fitzgibbon. Kinectfusion: Real-time dense surface mapping and tracking. In 2011 10th IEEE International Symposium on Mixed and Augmented Reality, pages 127-136, 2011.

[SWL $\left.{ }^{+} 16\right]$ Y. Sun, C. Wang, J. Li, Z. Zhang, D. Zai, P. Huang, and C. Wen. Automated segmentation of lidar point clouds for building rooftop extraction. In 2016 IEEE International Geoscience and Remote Sensing Symposium (IGARSS), pages 14721475, 2016.

[TBBS14] S. Tuttas, A. Braun, A. Borrmann, and U. Stilla. Comparision of photogrammetric point clouds with bim building elements for construction progress monitoring. volume 1, pages 341-345, 82014.

[TBBS17] S. Tuttas, A. Braun, A. Borrmann, and U. Stilla. Acquisition and consecutive registration of photogrammetric point clouds for construction progress monitoring using a $4 \mathrm{~d}$ bim. PFG - Journal of Photogrammetry, Remote Sensing and Geoinformation Science, 85(1):3-15, 2017.

[TC98] M. Teichmann and M. Capps. Surface reconstruction with anisotropic densityscaled alpha shapes. In Proceedings Visualization '98, pages 67-72, 1998.

[TSBB15] S. Tuttas, U. Stilla, A. Braun, and A. Borrmann. Validation of bim components by photogrammetric point clouds for construction site monitoring. ISPRS Annals of Photogrammetry, Remote Sensing \& Spatial Information Sciences, 2, 2015.

$\left[\mathrm{ZHK}^{+}{ }^{14}\right]$ S. Zollmann, C. Hoppe, S. Kluckner, C. Poglitsch, H. Bischof, and G. Reitmayr. Augmented reality for construction site monitoring and documentation. Proceedings of the IEEE special issue on applications of AR environments, 102:137154, 022014.

[ZLY19] W. Zhou, J. Lu, and W. Yue. A new semantic segmentation method of point cloud based on pointnet and voxelnet. In 2019 Chinese Control And Decision Conference (CCDC), pages 803-808, 2019. 